TRANSACTIONS OF THE

AMERICAN MATHEMATICAL SOCIETY

Volume 226, 1977

\title{
THE PERIODIC POINTS OF MORSE-SMALE ENDOMORPHISMS OF THE CIRCLE
}

BY

LOUIS BLOCK

\begin{abstract}
Let $M S\left(S^{1}\right)$ denote the set of continuously differentiable maps of the circle with finite nonwandering set, which satisfy certain generic properties. For $f \in M S\left(S^{1}\right)$ let $P(f)$ denote the set of positive integers which occur as the period of some periodic point of $f$. It is shown that for $f \in M S\left(S^{l}\right)$ there are integers $m \geqslant 1$ and $n>0$ such that $P(f)=\{m, 2 m$, $4 m, \ldots, 2^{n} m$ ). Conversely, if $m$ and $n$ are integers, $m>1, n>0$, there is a map $f \in M S\left(S^{1}\right)$ with $P(f)=\left\{m, 2 m, 4 m, \ldots, 2^{n} m\right\}$.
\end{abstract}

1. Introduction. This paper is concerned with determining the possible orbit structures for a certain set of differentiable maps of the circle. For an introduction to the general theory of the orbit structures of differentiable maps of manifolds see [8] and [9]. Other papers on differentiable maps of the circle include [3], [4], [5], [6], and [7].

We let $M S\left(S^{1}\right)$ denote the set of continuously differentiable maps $f$ of the circle to itself which satisfy the following properties (see $\$ 2$ for definitions):

(1) $\Omega(f)$ (the nonwandering set) is finite.

(2) All periodic points of $f$ are hyperbolic.

(3) No singularity of $f$ is eventually periodic.

It can be shown that these conditions imply the following (see [2]):

(4) $\Omega(f)$ is the set of periodic points of $f$.

In this paper we ask the following question. Let $f \in M S\left(S^{1}\right)$. What are the possible periods of the periodic points of $f$ ?

More precisely, we let $P(f)$ denote the finite set of positive integers which are the periods of periodic points of $f$ (so $n \in P(f)$ if and only if, for some $x \in S^{1}, f^{n}(x)=x$ and $\left.f^{k}(x) \neq x \forall k<n\right)$. We then ask what sets may occur as $P(f)$ for $f \in M S\left(S^{1}\right)$.

The following theorem which we proved in [2] gives a partial answer.

THEOREM A. Let $f \in M S\left(S^{1}\right)$. There is a natural number $n(f)$ such that the period of any periodic point of $f$ is $n(f)$ times a power of 2 .

Received by the editors April 28, 1975 and, in revised form, October 6, 1975.

AMS (MOS) subject classifications (1970). Primary 58F15.

Key words and phrases. Endomorphism, nonwandering set, periodic point. 
Theorem A implies for example that $f \in M S\left(S^{1}\right)$ cannot have a fixed point and a periodic point of period 3. However, Theorem $\mathrm{A}$ does not answer questions like the following: If $f \in M S\left(S^{1}\right)$ has a fixed point and a periodic point of period 4, must $f$ have a periodic point of period 2?

The main results of this paper are the following two theorems which completely answer the question of what sets may occur as $P(f)$ for $f$ $\in M S\left(S^{1}\right)$.

TheOREM B. Let $f \in M S\left(S^{1}\right)$. There are integers $m$ and $n, m \geqslant 1, n \geqslant 0$, such that $P(f)=\left\{m, 2 m, 4 m, \ldots, 2^{n} m\right\}$.

THEOREM C. Let $m$ and $n$ be integers, $m \geqslant 1, n \geqslant 0$. There is a map $f \in M S\left(S^{1}\right)$ with $P(f)=\left\{m, 2 m, 4 m, \ldots, 2^{n} m\right\}$.

We close this section by remarking that properties (2) and (3) of the definition of $M S\left(S^{1}\right)$ are generic, i.e., true for a Baire subset of $C^{1}\left(S^{1}, S^{1}\right)$ (the space of continuously differentiable maps of the circle to itself with the $C^{1}$ topology). Also for $f \in M S\left(S^{1}\right)$ to be structurally stable we need two other technical conditions in addition to the ones given here (see [2]). However, $M S\left(S^{1}\right)$ as defined here has the property (easily checked) that if $f \in M S\left(S^{1}\right)$ then $f^{n} \in M S\left(S^{1}\right)$ for any positive integer $n$, and this property would not be true if we required $f \in M S\left(S^{1}\right)$ to satisfy the two additional conditions necessary for structural stability.

2. Preliminary definitions and results. Let $f \in C^{1}\left(S^{1}, S^{1}\right)$. A point $x \in S^{1}$ is said to be wandering if there is a neighborhood $V$ of $x$ with $f^{n}(V) \cap V$ $=\varnothing$ for all positive integers $n$. The set of points on the circle which are not wandering is called the nonwandering set and denoted $\Omega(f)$.

A point $x \in S^{1}$ is called a singularity of $f$ if $D f(x)=0$ where $D f(x)$ denotes the derivative of $f$ at $x$. $x$ is called a periodic point of $f$ if $f^{n}(x)=x$ for some positive integer $n . x$ is said to be eventually periodic if $f^{k}(x)$ is a periodic point for some positive integer $k$.

Let $x \in S^{1}$ be a periodic point of $f$ of period $n$. We say $x$ is expanding if $\left|D f^{n}(x)\right|>1$, and contracting if $\left|D f^{n}(x)\right|<1$. We say $x$ is hyperbolic if it is either expanding or contracting.

We will use the notation $(a, b)$ to denote the open arc from $a$ counterclockwise to $b$, and $[a, b]$ to denote the closed arc from $a$ counterclockwise to $b$. We will say periodic points $x$ and $y$ are adjacent periodic points if there aie no periodic points in one of the intervals $(x, y),(y, x)$.

We state the following two lemmas which are proved in [2]. In Lemma 2, $\Omega_{e}(f)$ denotes the set of expanding periodic points of $f$, and $\Omega_{c}(f)$ denotes the set of contracting periodic points of $f$.

Lemma 1. Let $f \in M S\left(S^{1}\right)$. Suppose $e$ and $c$ are adjacent periodic points of $f$ 
with e expanding and $c$ contracting. If $c$ is a fixed point of $f$ then $e$ is fixed by $f^{2}$.

LEMMA 2. Let $f \in M S\left(S^{1}\right)$. If $f$ is onto, then cardinality $\Omega_{e}(f)=$ cardinality $\Omega_{c}(f)$ and the expanding and contracting periodic points alternate. If $f$ is not onto, then cardinality $\Omega_{c}(f)=$ cardinality $\Omega_{e}(f)+1$.

In this case (if $f$ has more than one periodic point) there is one pair of adjacent contracting periodic points, but otherwise the expanding and contracting periodic points alternate. Furthermore if $x$ and $y$ are the two adjacent contracting periodic points, with no periodic points in the open interval $(x, y)$ then $(x, y)$ is not contained in $f\left(S^{1}\right)$.

Let $f \in C^{1}\left(S^{1}, S^{1}\right)$. For a subset $A \subset S^{1}$, let orb $(A)$ denote $\cup_{n \geq 0} f^{n}(A)$.

Let $e$ be an expanding fixed point of $f$. Let $U$ be any open interval about $e$ with $|D f(x)|>1 \forall x \in U$. Let $W^{u}(e)=$ orb $(U)$. Then $W^{u}(e)$ is clearly well defined. If $e$ is orientation preserving (i.e. if $D f(e)>0)$, we let $W^{u}(e, c c)$ denote orb $([e, b])$ where $b$ is any point with $|D f(x)|>1 \forall x \in[e, b]$. Again $W^{u}(e, c c)$ is well defined. If $e$ is orientation reversing we define $W^{u}(e, c c)$ by thinking of $e$ as an orientation preserving fixed point of $f^{2}$.

Let $c$ be a contracting fixed point of $f$. Let $W^{s}(c)$ (the stable manifold of $c$ ) denote the set of $x \in S^{1}$ such that $c$ is a limit point of $\operatorname{orb}(x)$. We let slsm $(c)$ denote the component of $W^{s}(c)$ which contains $c$. If $c$ is a contracting periodic point of period $n$, we define $W^{s}(c)$ and $s / s m(c)$ by thinking of $c$ as a fixed point of $f^{n}$.

The following two lemmas can be easily proved (see [2]). The proof of Lemma 3 uses Lemma 6 below.

LEMMA 3. Let $e$ be an expanding orientation preserving fixed point of $f \in M S\left(S^{1}\right)$. Let I denote the closure of $W^{u}(e, c c)$. Then I is a proper closed subinterval of $S^{1}$ and $f$ has another fixed point in $I$ (in addition to $e$ ).

LEMMA 4. Suppose $c$ is a contracting fixed point of $f \in M S\left(S^{\prime}\right)$, and $W^{s}(c) \neq S^{1}$. If $e_{1}$ and $e_{2}$ are the endpoints of the open interval slsm(c), then one of the following must occur:

(1) $e_{1}$ and $e_{2}$ are expanding fixed points.

(2) $e_{1}$ and $e_{2}$ are expanding periodic points of period 2.

(3) $e_{1}$ is an expanding fixed point and $f\left(e_{2}\right)=e_{1}$.

(4) $e_{1}=e_{2}$ is an expanding fixed point.

The following lemma follows immediately by continuity (the intermediate value theorem).

Lemma 5. Suppose $f$ is a continuous map of the circle to itself. Suppose $K$ is a closed interval on $S^{1}$ with $f(K) \supset K$ and $f(K) \neq S^{1}$. Then $f$ has a fixed point in $K$. 
We conclude this section with the following easy lemma which is one of the main consequences of the condition that $\Omega(f)$ is finite.

LeMMA 6. Let $f \in M S\left(S^{1}\right)$ and let $e$ be an expanding fixed point of $f$. If $x$ is in the closure of $W^{u}(e)$ and $x \neq e$, then $f(x) \neq e$.

Proof. If $x \in \overline{W^{u}(e)}$ and $x \neq e$, and $f(x)=e$, then $x$ is nonwandering but not periodic contradicting property (4) of $M S\left(S^{1}\right)$ (see $\left.\S 1\right)$. Here we have used property (3) to insure that for any neighborhood $V$ of $x, f(V)$ contains a neighborhood of $e$. Q.E.D.

3. Proof of Theorem B. In following many of the proofs in this section it may be helpful for the reader to draw a circle and label important points on the circle in the correct order.

LEMMA 7. Suppose $f \in M S\left(S^{1}\right)$ and $f$ has a fixed point, and a periodic point of period $n \geqslant 4$, but no periodic points of period 2. Then $f$ has an expanding fixed point adjacent to a contracting periodic point of period $k \geqslant 4$.

Proof. Clearly $f$ must have adjacent periodic points $p$ and $q$ with $p$ fixed and $q$ of period at least 4 . We have the following cases:

Case 1. Both $p$ and $q$ are contracting.

Case 2. Both $p$ and $q$ are expanding.

Case 3. $p$ is contracting and $q$ is expanding.

Case 4. $q$ is contracting and $p$ is expanding.

By Lemma 2, Case 2 is impossible, and by Lemma 1, Case 3 is impossible. In Case 4, the lemma is proved, so it suffices to look at Case 1.

Suppose $p$ and $q$ are contracting. Without loss of generality we may assume that there are no periodic points in $(q, p)$. Then there must be adjacent periodic points $a$ and $b$ in the closed interval $[p, q]$ with $a$ fixed and $b$ of period at least 4 , and $\{a, b\} \neq\{p, q\}$. As above we have the same four possible cases (with Case 2 and Case 3 impossible), but now by Lemma 2, $a$ and $b$ cannot both be contracting. Hence the only possibility is that $a$ is expanding and $b$ is contracting. Q.E.D.

LeMma 8. Let $f \in C^{0}\left(S^{1}, S^{1}\right)$. Let $A$ and $B$ be proper closed intervals of $S^{1}$ with $f(A) \supset B$ and $f(A) \neq S^{1}$. There is a closed interval $J \subset A$ with $f(J)=B$.

Proof. Let $B=\left[b_{1}, b_{2}\right]$. Since $f^{-1}\left(b_{1}\right) \cap A$ and $f^{-1}\left(b_{2}\right) \cap A$ are disjoint compact sets, there are points $v \in f^{-1}\left(b_{1}\right) \cap A$ and $w \in f^{-1}\left(b_{2}\right) \cap A$ such that if $W$ is the open interval joining $v$ and $w$ with $W \subset A$ then $W$ $\cap\left(f^{-1}\left(b_{1}\right) \cup f^{-1}\left(b_{2}\right)\right)=\varnothing$.

Let $J$ be the closure of $W$. $f(J)$ must contain either $B$ or the interval $\left[b_{2}, b_{1}\right]$. Since $f(A) \neq S^{1}$ but $f(A) \supset B, f(J)$ does not contain the interval $\left[b_{2}, b_{1}\right]$. Hence $f(J) \supset B$. Since no points in the interior of $J$ are mapped to an endpoint of $B$ we have $f(J)=B$. Q.E.D. 
Lemma 9. Let $f \in C^{0}\left(S^{1}, S^{1}\right)$. Suppose $I_{1}, \ldots, I_{k}$ are proper closed intervals of $S^{1}$ with disjoint interiors, with the property that $f\left(I_{j}\right) \supset I_{j+1}$ for $j=1, \ldots$, $k-1$ and $f\left(I_{k}\right) \supset I_{1}$. Suppose also that $f\left(I_{j}\right) \neq S^{1}$ for $j=1, \ldots, k$. Finally, suppose that for all $r \in\{1, \ldots, k-1\}$, neither endpoint of $I_{1}$ is a periodic point of $f$ of period $r$. Then $f$ has a periodic point of period $k$ in $I_{1}$.

Proof. By Lemma 8, there are closed intervals $J_{1}, \ldots, J_{k}$ such that $J_{i} \subset I_{i}$ for $i=1, \ldots, k$ and $f\left(J_{k}\right)=I_{1}$ while $f\left(J_{i}\right)=J_{i+1}$ for $i=1, \ldots, k-1$. It follows that $f^{k}\left(J_{1}\right)=I_{1}$. By Lemma 5 , there is a fixed point $p$ of $f^{k}$ in $J_{1} \subset I_{1}$.

If $p$ is an endpoint of $I_{1}$, then, by hypothesis, $p$ is a periodic point of $f$ of period $k$. If $p$ is an interior point of $I_{1}$, then since $f^{i}(p) \in J_{i+1} \subset I_{i+1}$ for $i=1, \ldots, k-1$ and the intervals $I_{j}$ have disjoint interior, $p$ must be a periodic point of $f$ of period $k$. Q.E.D.

LEMMA 10. Suppose $f \in M S\left(S^{1}\right)$ and f has an expanding fixed point e adjacent to a periodic point $k_{1}$ of period $n \geqslant 4$. If $e$ is orientation preserving then $f$ has a periodic point of period 2.

Proof. Without loss of generality we may assume that there are no periodic points in $\left(e, k_{1}\right)$ (the open arc from $e$ counterclockwise to $k_{1}$ ).

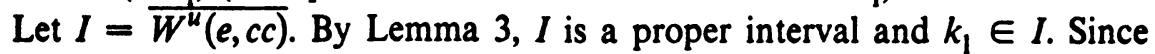
$f(I)=I$, we have orb $\left(k_{1}\right) \subset I$. Let orb $\left(k_{1}\right)=\left\{k_{1}, k_{2}, k_{3}, \ldots, k_{n}\right\}$ where we number these points in order counterclockwise around the circle beginning with $k_{1}$.

Consider the $n-1$ closed intervals, $\left[k_{1}, k_{2}\right],\left[k_{2}, k_{3}\right], \ldots,\left[k_{n-1}, k_{n}\right]$. Note that each of these intervals is contained in $I$, and $f(I)=I$. Hence for each $i=1, \ldots, n-1, f\left(\left[k_{i}, k_{i+1}\right]\right)$ contains at least one interval $\left[k_{j}, k_{j+1}\right]$ with $j \neq i$. It follows (using Lemma 9) that $f$ has a periodic point of period $r_{1}$ in $I$, with $2 \leqslant r_{1} \leqslant n-1$.

Repeating the argument (of the last paragraph) $n-3$ times with the new periodic point in place of $k_{1}$, we see that $f$ has a periodic point of period 2 . Q.E.D.

LEMMA 11. Let $f \in M S\left(S^{1}\right)$. Suppose $e_{1}$ and $e_{2}$ are distinct expanding fixed points of $f$, and $\overline{W^{u}\left(e_{1}\right)}=S^{1}$ (where $\overline{W^{u}\left(e_{1}\right)}$ denotes the closure of $\left.W^{u}\left(e_{1}\right)\right)$. Then $\overline{W^{u}\left(e_{2}\right)} \neq S^{1}$.

Proof. Since $\overline{W^{u}\left(e_{1}\right)}=S^{1}$, it follows from Lemma 6 that if $f(x)=e_{1}$ then $x=e_{1}$.

Let $I$ be an open interval containing $e_{1}$ with $|D f(x)|>I \forall x \in I$, and the length of $f(I)$ less than the distance from $e_{1}$ to $e_{2}$. Let $J=S^{1}-f\left(S^{1}-I\right)$. Then $J$ is an open interval containing $e_{1}$, because $f\left(S^{\prime}-I\right)$ is compact and does not contain $e_{1}$.

Let $K=(a, b)$ be an open interval containing $e_{1}$ with the following properties: 
(1) $a$ and $b$ are the same distance from $e_{1}$.

(2) $K \subset J \cap I$.

(3) $e_{2}$ is not in the closure of $K$. (This actually follows from (2).)

We claim $f\left(S^{1}-K\right) \subset S^{1}-K$. To prove this let $x \in S^{1}-K$. First suppose $x \notin I$. Then $f(x) \in f\left(S^{1}-I\right)$. Hence $f(x) \notin J$ so $f(x) \in S^{1}-K$. Now suppose $x \in I$. Then the distance from $f(x)$ to $e_{1}$ must be greater than the distance from $x$ to $e_{1}$. Since $x \in S^{1}-K$, we have $f(x) \in S^{1}-K$. This proves the claim.

Since $e_{2}$ is an interior point of $S^{1}-K$ and $f\left(S^{1}-K\right) \subset S^{1}-K$, it follows that $W^{u}\left(e_{2}\right) \subset S^{1}-K$. Hence $\overline{W^{u}\left(e_{2}\right)} \neq S^{1}$. Q.E.D.

LEMMA 12. Suppose $f \in M S\left(S^{1}\right)$ has a fixed point, and a periodic point of period at least 4 , and no periodic points of period 2. Then $f$ has an orientation reversing expanding fixed point e with $\overline{W^{u}(e)} \neq S^{1}$.

Proof. By Lemma $7, f$ must have an expanding fixed point $e_{0}$ adjacent to a contracting periodic point $k$ of period at least 4. By Lemma $10, e_{0}$ is orientation reversing. We may assume without loss of generality that there are no periodic points in $\left(e_{0}, k\right)$. Also we may assume that $\overline{W^{u}\left(e_{0}\right)}=S^{1}$ (or else we are done).

We claim that $f$ has an expanding fixed point $e_{1} \neq e_{0}$. To prove this claim let $I=\overline{W^{u}\left(e_{0}, c c\right)}$. Then $I$ is a proper closed interval and $f^{2}(I) \subset I$. It follows from Lemma 3 that $f^{2}$ has another fixed point $c \in I\left(c \neq e_{0}\right)$. Since $f$ has no periodic points of period $2, c$ is a fixed point of $f$.

If $c$ is expanding the claim is proven, so we may assume that $c$ is contracting. By Lemma 4, and the fact that $f$ has no periodic points of period 2, it follows that one endpoint $e_{2}$ of $s l s m(c)$ is an expanding fixed point. If $e_{2} \neq e_{0}$ the claim is proven.

Suppose $e_{2}=e_{0}$, i.e., $e_{0}$ is an endpoint of $s / s m(c)$. Let $e_{3}$ denote the other endpoint of $\operatorname{slsm}(c)$ (i.e., $e_{3} \neq e_{0}$ ). Then since $\overline{W^{u}\left(e_{0}\right)}=s^{l}$, by Lemma 6 we have $f\left(e_{3}\right) \neq e_{0}$. Hence $e_{3}$ is an expanding fixed point by Lemma 4. This proves the claim that $f$ has an expanding fixed point $e_{1} \neq e_{0}$.

In $\left[e_{0}, e_{1}\right]$ there is a point $k$ of period at least 4 and the fixed point $e_{1}$. Hence in the interval $\left[k, e_{1}\right]$ there must be adjacent periodic points $c_{1}$ and $e$ with $c_{1}$ of period at least 4 and $e$ fixed. Since $\overline{W^{u}\left(e_{0}\right)}=S^{1}$ implies that $f$ is onto, it follows from Lemmas 1 and 2 that $c_{1}$ is contracting and $e$ is expanding. By Lemma $10, e$ is orientation reversing. Since $\overline{W^{u}\left(e_{0}\right)}=S^{1}$ and $e \neq e_{0}$, by Lemma 11 we have $\overline{W^{u}(e)} \neq S^{1}$. Q.E.D.

THEOREM 13. Let $f \in M S\left(S^{1}\right)$. Suppose $f$ has a fixed point and a periodic point of period at least 4 . Then $f$ has a periodic point of period 2 .

Proof. By Lemma 12, we may assume that $f$ has an orientation reversing expanding fixed point $e$ such that $\overline{W^{u}(e)} \neq S^{1}$. Let $\overline{W^{u}(e)}=[a, b]$. Note that 
$f$ maps the interval $[a, e]$ onto the interval $[e, b]$, and $f$ maps the interval $[e, b]$ onto the interval $[a, e]$. This is true because $f$ maps the intervai $[a, b]$ onto itself, $f$ is orientation reversing at $e$, and for $x \in[a, b]$, if $x \neq e$ then $f(x) \neq e$ by Lemma 6.

Pick $k_{1} \in(e, b)$ so that for all $k \in\left(e, k_{1}\right), f^{2}(k) \neq k$ and $\left(k, f^{2}(k)\right) \subset(e, b)$ (using the fact that $e$ is an expanding fixed point). Let $\left[k_{2}, r\right]$ denote the image under $f^{2}$ of $\left[k_{1}, b\right]$. Then $k_{2} \in(e, b)$. Let $k_{0} \in\left(e, k_{1}\right) \cap\left(e, k_{2}\right)$ and let $I=\left[k_{0}, b\right]$. Then $f^{2}(I) \subset I$. Hence $f^{2}$ has a fixed point $y \in I$. But $f(I) \cap I$ $=\varnothing$. Hence $y$ is a periodic point of $f$ of period 2. Q.E.D.

LEMMA 14. Let $f \in M S\left(S^{1}\right)$. Suppose f has periodic points of periods $k$ and $m$ respectively with $k<m$. Then $k$ divides $m$.

Proof. This follows immediately from Theorem A of $\$ 1$ which is proved in [2]. Q.E.D.

THEOREM 15. Let $f \in M S\left(S^{1}\right)$. Suppose $f$ has a periodic point of period $m$ and a periodic point of period $2^{n} m$ where $n>1$. Then $f$ has a periodic point of period $2 m$.

Proof. Let $g=f^{m}$. Then $g \in M S\left(S^{1}\right)$ and $g$ has a fixed point and a periodic point of period $2^{n}$. By Theorem $13, g$ has a periodic point $x$ of period 2. So $g^{2}(x)=x$ and $g(x) \neq x$. This implies $f^{2 m}(x)=x$ and $f^{m}(x) \neq x$.

Let $k$ be the period of $x$ as a periodic point of $f$. Then $k$ divides $2 m$, but $k$ does not divide $m$. By Lemma $14, k=2 m$. Q.E.D.

We can now easily prove Theorem B. Recall that $P(f)$ denotes the set of positive integers which occur as the period of some periodic point of $f$.

THEOREM B. Let $f \in M S\left(S^{1}\right)$. There are integers $m$ and $n, m \geqslant 1, n \geqslant 0$, such that

$$
P(f)=\left\{m, 2 m, 4 m, \ldots, 2^{n} m\right\} .
$$

Proof. Let $m$ be the smallest period of any periodic point of $f$. By Theorem A (see $\$ 1$ ) the largest period of any periodic point of $f$ is $2^{n} m$ for some nonnegative integer $n$. By Theorem A, $P(f) \subset\left\{m, 2 m, 4 m, \ldots, 2^{n} m\right\}$. But $m \in P(f)$ and $2^{n} m \in P(f)$ so by repeated application of Theorem 15 we have

$$
P(f)=\left\{m, 2 m, 4 m, \ldots, 2^{n} m\right\} .
$$

Q.E.D.

4. Proof of Theorem C. A major part of the proof of Theorem $\mathrm{C}$ is contained in the following lemma. We use the notation $\Omega_{e}(g)$ to denote the set of expanding periodic points of $g$. 
LEMMA 16. Let I be a proper closed interval of $S^{1}$. For any natural number $n \geqslant 2$, there is a continuously differentiable map $g_{n}$ from I into itself with the following properties:

(1) $g_{n}$ has exactly one singularity $t$ in the interior of $I$.

(2) $t$ is a periodic point of $g_{n}$ of period $2^{n}$.

(3) $g_{n}$ has exactly one expanding fixed point $e$, and $g_{n}$ is orientation reversing at $e$.

(4) $\Omega_{e}\left(g_{n}\right)$ consists of $n$ periodic orbits of period $1,2,4, \ldots, 2^{n-1}$ respectively.

(5) $\Omega\left(g_{n}\right)=\operatorname{orb}(t) \cup \Omega_{e}\left(g_{n}\right)$.

(6) The (one-sided) derivative of $g_{n}$ is zero at the endpoints of $I$.

Proof. The proof is by induction. For $n=2$, the map $g_{2}$ is constructed as in Figure 1. We use the notation $t^{k}$ to denote $\left(g_{2}\right)^{k}(t)$ where $k$ is a positive integer. In Figure 1 , since $g$ permutes the intervals $\left[t^{1}, t^{3}\right]$ and $\left[t^{4}, t^{2}\right]$, there are periodic points $e_{1}$ and $e_{2}$ of period 2 in these intervals. We can clearly arrange that $\Omega_{e}\left(g_{2}\right)=\left\{e, e_{1}, e_{2}\right\}$ and $\Omega\left(g_{2}\right)=\Omega_{e}\left(g_{2}\right) \cup$ orb $(t)$, and that property (6) is satisfied.

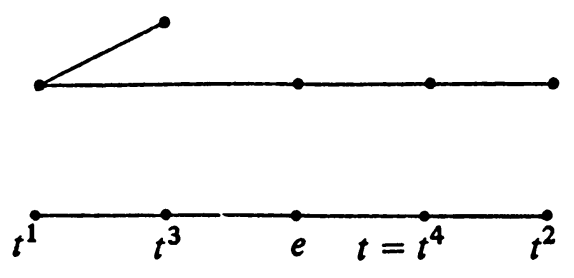

FIGURE 1

The map $g_{2}$

Proceeding now by induction we assume $g_{n}$ is defined satisfying properties (1)-(6). We suppose we have drawn a diagram of $g_{n}$ labeling the iterates of $t$, as we did with $g_{2}$ in Figure 1 . We will modify this diagram of $g_{n}$ and show that there is a map $g_{n+1}$ corresponding to the modified diagram which satisfies properties (1)-(6).

Let $k_{1}, k_{2}, \ldots, k_{\left(2^{n}\right)}$ denote the powers of $t\left(1 \leqslant k_{i} \leqslant 2^{n}\right)$ on the diagram of $g_{n}$ in order from left to right. For example, if $g_{n}=g_{2}$ then $k_{1}=1, k_{2}=3$, $k_{3}=4$, and $k_{4}=2$, since $t^{1}, t^{3}, t^{4}$, and $t^{2}$ are the iterates of $t$ in order from left to right on the diagram of $g_{2}$. By induction we may assume that $\left|k_{i}-k_{i+1}\right|=2^{n-1}$ for $i$ odd.

Now in each of the intervals $\left[t^{\left(k_{i}\right)}, t^{\left(k_{i+1}\right)}\right]$ where $i$ is odd we add two points $t^{\left(l_{i}\right)}$ and $t^{\left(l_{i+1}\right)}$, with $t^{\left(l_{i}\right)}$ to the left of $t^{\left(l_{i+1}\right)}$, where $l_{i}=k_{i}+2^{n}$ and $l_{i+1}$ $=k_{i+1}+2^{n}$. Then at the point $t^{\left(2^{n+1}\right)}$ we write $t=t^{\left(2^{n+1}\right)}$. Note that for the value of $i$ with $k_{i}=2^{n}$ we have $l_{i}=2^{n}+2^{n}=2^{n+1}$. 
It should be noted here that we are using the points $t^{\left(l_{i}\right)}$ and $t^{\left(k_{i}\right)}$ to describe the orbit of a new singularity $t$ of $g_{n+1}$. A good way to think of what we have done is the following. Let $s=2^{n}$. Relabel the points $t^{1}, t^{2}, \ldots, t^{s}$ as $p_{1}, p_{2}, \ldots, p_{s}$. For $g_{n}, t^{s}=t$ is its singularity. Near each $p_{i}$ we pick a point $q_{i}$ (on the appropriate side of $p_{i}$ ). We then modify the map $g_{n}$ so that for the new map $g_{n+1}$ the point $q_{s}$ is a singularity and $g_{n+1}\left(p_{s}\right)=q_{1}$ while $g_{n+1}\left(q_{s}\right)=p_{1}$. Thus the successive iterates of $q_{s}$ under $g_{n+1}$ are $q_{s}, p_{1}, p_{2}, \ldots, p_{s}, q_{1}, q_{2}, \ldots$, $q_{s}, \ldots$. Finally we relabel $t=q_{s}, t^{1}=p_{1}, t^{2}=p_{2}$, etc. Then $t$ is a singularity of $g_{n+1}$ of period $2 s=2^{n+1}$.

This is the modified diagram we want. By construction and our induction hypothesis it follows that there is a map $g_{n+1}$ with iterates of $t$ as described in the modified diagram. (It may be helpful to see Figure 2 in which the diagram of $g_{3}$ is given, and compare with the diagram of $g_{2}$ in Figure 1.)

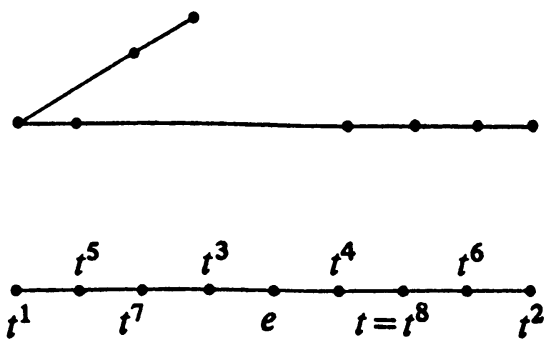

FIGURE 2

The map $g_{3}$

By construction, $g_{n+1}$ satisfies property (2), and we can easily insure that properties (1), (3), and (6) are also satisfied. We must show $g_{n+1}$ can be constructed to also satisfy properties (4) and (5).

Consider the $2^{n}$ intervals of the form $\left[t^{\left(k_{i}\right)}, t^{\left(l_{i}\right)}\right]$ with $i$ odd or $\left[t^{\left(l_{i}\right)}, t^{\left(k_{i}\right)}\right]$ with $i$ even, where $k_{i}=1, \ldots, 2^{n}$. Since $l_{i}=k_{i}+2^{n}$ it follows that these intervals are permuted by $g_{n+1}$. Hence we can arrange that in each of these intervals there is an expanding periodic point of period $2^{n}$, with everything else in these intervals in the stable manifold of the orbit of $t$.

Let $K$ be the union of the $2^{n-1}$ intervals of the form $\left[t^{\left(k_{i}\right)}, t^{\left(k_{i+1}\right)}\right]$, $i$ odd, $i=1,3, \ldots, 2^{n}-1$. Then by construction, $g_{n+1}(K)=K$, and we can label the intervals $\left[t^{\left(k_{i}\right)}, t^{\left(k_{i+1}\right)}\right]$ as $K_{1}, K_{2}, \ldots, K_{\left(2^{n-1}\right)}$ where $g_{n+1}\left(K_{i}\right)=K_{i+1}$ for $i=1, \ldots, 2^{n-1}-1$ and $g_{n+1}\left(K_{i}\right)=K_{1}$ for $i=2^{n-1}$.

Now consider the $2^{n-1}$ intervals of the form $\left[t^{\left(l_{i}\right)}, t^{\left(l_{i+1}\right)}\right]$ where $i$ is odd, $i=1,3, \ldots, 2^{n}-1$. Let $L$ denote the union of these intervals. Note that $\left[t^{\left(l_{i}\right)}, t^{\left(l_{i+1}\right)}\right] \subset\left[t^{\left(k_{i}\right)}, t^{\left(k_{i+1}\right)}\right]$ (for $i$ odd) and

$$
\left(g_{n+1}\right)^{\left(2^{n-1}\right)}\left(\left[t^{\left(l_{i}\right)}, t^{\left(l_{i+1}\right)}\right]\right) \supset\left[t^{\left(l_{i}\right)}, t^{\left(l_{i+1}\right)}\right] .
$$


Hence we can arrange that in each interval $\left[t^{\left(i_{1}\right)}, t^{\left(l_{i+1}\right)}\right]$ there is an expanding periodic point of period $2^{n-1}$, and all other interior points of $L$ are wandering.

So far we have shown that we can construct $g_{n+1}$ on the set $K$ so that $g_{n+1}(K) \subset K$ and $\Omega\left(g_{n+1} \mid K\right)$ consists of three periodic orbits, namely expanding periodic orbits of period $2^{n}$ and $2^{n-1}$, and the orbit of the singularity $t$ of period $2^{n+1}$.

Let $B$ be the closure of an interval in $I-K$. Then for each $k>0,\left(g_{n}\right)^{k}(B)$ $\supset B$ if and only if $\left(g_{n+1}\right)^{k}(B) \supset B$. Hence we can arrange that on $I-K, g_{n+1}$ (like $g_{n}$ ) has $n-1$ expanding periodic orbits of period $2^{n-2}, 2^{n-3}, \ldots, 2,1$, with everything else in $I-K$ wandering. Thus $g_{n+1}$ satisfies properties (1)-(6). Q.E.D.

We state the following easy lemma which is a special case of the more general $\Omega$-stability theorems of [1] or [5] (a similar theorem may be found in [7]). Note that $f$ may have a singularity which is also a contracting periodic point and still satisfy the hypothesis of the lemma.

LEMma 17. Suppose $f \in C^{1}\left(S^{1}, S^{1}\right)$ and $f$ satisfies the following:

(1) $\Omega(f)$ is a finite set of periodic points.

(2) All periodic points of $f$ are hyperbolic.

(3) All singularities of $f$ are in the stable manifolds of contracting periodic points.

Then for $g$ sufficiently close to in $C^{1}\left(S^{1}, S^{1}\right), g$ will also satisfy conditions (1), (2), and (3). Furthermore, for each positive integer $k$, there will be a one-to-one correspondence between the periodic points of $f$ and $g$ of period $k$, with expanding (respectively, contracting) periodic points of $f$ corresponding to expanding (respectively, contracting) periodic points of $g$.

For an interval $J$ of $S^{1}$ we define $M S(J)$ to be the set of continuously differentiable maps of $J$ into itself which satisfy the same properties as in the definition of $M S\left(S^{1}\right)$ in $\$ 1$.

LEMMA 18. Let $J$ be a proper closed interval of $S^{1}$. For any natural number $n$, $\exists f_{n} \in M S(J)$ such that:

(1) $P\left(f_{n}\right)=\left\{1,2,4, \ldots, 2^{n}\right\}$.

(2) The endpoints of $J$ are expanding fixed points of $f_{n}$.

Proof. Let $I=[a, b]$ be a proper subinterval of $J$. For $n \geqslant 2$, let $g_{n}$ be defined on $I$ as in Lemma 16 . For $n=1$, let $g_{1}$ be a continuously differentiable map of $I$ onto itself with $\Omega\left(g_{1}\right)=\{a, b, e\}$ where $e$ is an orientation reversing expanding fixed point of $g_{1}, a$ and $b$ are contracting periodic points of period two with $D g_{1}(a)=D g_{1}(b)=0$, and $a$ and $b$ are the only singularities of $g_{1}$.

Let $J=[c, d]$. We extend $g_{n}$ to $J$ as follows. Let $g_{n}$ map the interval $[c, a]$ onto the interval $\left[c, g_{n}(a)\right]$ so that:

(A) $c$ is an expanding fixed point of $g_{n}$. 
(B) For each $x \in(c, a), g_{n}(x)$ is to the right of $x$ in $[c, d]$.

(C) $g_{n}$ is continuously differentiable on $[c, a]$.

(D) $D g_{n}(a)=0$ and $D g_{n}(x) \neq 0$ if $x \in(c, a)$.

We define $g_{n}$ similarly on $[b, d]$. Then $g_{n}$ is a continuously differentiable map of $J$ onto itself, $\Omega\left(g_{n}\right)$ is a finite set of periodic points, and $g_{n}$ satisfies properties (1) and (2) of the conclusion of the lemma.

By construction, all periodic points of $g_{n}$ are expanding except for one contracting periodic orbit which contains all singularities of $g_{n}$. We can easily perturb $g_{n}$ to a map $f_{n}$ (i.e. there is a map, $f_{n}$, arbitrarily close to $g_{n}$ in $C^{1}(J, J)$ ) for which this periodic orbit is replaced by the orbit of a contracting periodic point $k$, with no singularities in $\operatorname{orb}(k)$. By Lemma 17 , we can make the perturbation small enough to insure that $\Omega\left(f_{n}\right)$ consists of periodic points, the periodic points of $f_{n}$ correspond to those of $g_{n}$, and all singularities of $f_{n}$ are in the stable manifolds of points in the orbit of $k$. Thus $f_{n} \in M S(J)$ and $f_{n}$ satisfies (1) and (2). Q.E.D.

THEOREM C. Let $m$ and $n$ be integers, $m \geqslant 1, n \geqslant 0$. There is a map $f \in M S\left(S^{1}\right)$ with $P(f)=\left\{m, 2 m, 4 m, \ldots, 2^{n} m\right\}$.

Proof. For $n=0$ the theorem is obvious so we may assume $n \geqslant 1$. Let $I_{1}, \ldots, I_{m}$ be disjoint proper closed intervals of $S^{1}$, numbered in order around the circle. For $k=1, \ldots, m-1$, let $f$ be any orientation preserving diffeomorphism from $I_{k}$ onto $I_{k+1}$. Then define $f$ on $I_{m}$ by $f=f_{n} \circ f^{-(m-1)}$ where $f_{n}: I_{1} \rightarrow I_{1}$ is the map defined in Lemma 18 , and $f^{-(m-1)}$ makes sense as $f$ has been defined on $I_{1}, I_{2}, \ldots, I_{m-1}$.

Let $I_{k}=\left[a_{k}, b_{k}\right]$. Then for $k=1, \ldots, m-1, f\left(a_{k}\right)=a_{k+1}$ and $f\left(b_{k}\right)$ $=b_{k+1}$ while $f\left(a_{m}\right)=a_{1}$ and $f\left(b_{m}\right)=b_{1}$. Also, each of the sets $\left\{a_{1}, \ldots, a_{m}\right\}$ and $\left\{b_{1}, \ldots, b_{m}\right\}$ form an expanding periodic orbit. Hence we can extend $f$ to a continuously differentiable map of the circle with a contracting periodic orbit of period $m$ in $S^{1}-\left(I_{1} \cup \cdots \cup I_{m}\right)$ and all other points of $S^{1}$ $-\left(I_{1} \cup \cdots \cup I_{m}\right)$ wandering.

Then $f \in M S\left(S^{1}\right)$ and $P(f)=\left\{m, 2 m, 4 m, \ldots, 2^{n} m\right\}$. Q.E.D.

Finally, we remark that it is clear from the construction of the map $f$ in Theorem $\mathrm{C}$, that $f$ can be made to be $C^{\infty}$.

\section{REFERENCES}

1. L. Block, Diffeomorphisms obtained from endomorphisms, Trans. Amer. Math. Soc. 214 (1975), 403-413.

2. - Morse-Smale endomorphisms of the circle, Proc. Amer. Math. Soc. 48 (1975), 457-463.

3. L. Block and J. Franke, $A$ classification of the structurally stable contracting endomorphisms of $S^{1}$, Proc. Amer. Math. Soc. 36 (1972), 597-602. MR 46 \#264.

4. Existence of periodic points for maps of $S^{\prime}$, Invent. Math. 22 (1973/74), 69-73. MR $50 \# 11326$. 
5. M. V. Jakobson, On smooth mappings of the circle into itself, Mat. Sb. (N.S.) 85 (127) (1971), 163-188 = Math. USSR Sbornik 14 (1971), 161-185. MR 44 \# 7587.

6. Z. Nitecki, Factorization of non-singular circle endomorphisms, Dynamical Systems (Proc. Sympos., Univ of Bahia, Salvador, Brasil, 1971), Academic Press, New York, 1973.

7. - Partitions for circle endomorphisms, Dynamical Systems (Proc. Sympos., Univ. of Bahia, Salvador, Brasil, 1971), Academic Press, New York, 1973.

8. M. Shub, Endomorphisms of compact differentiable manifolds, Amer. J. Math. 91 (1969), 175-199. MR 39 \#2169.

9. S. Smale, Differentiable dynamical systems, Bull. Amer. Math. Soc. 73 (1967), 747-817. MR 37 \#3598; erratum, 39, p. 1593.

Department of Mathematics, University of Florida, Gainesville, Florida 32611 